\title{
4DCT and CBCT based PTV margin in Stereotactic Body Radiotherapy(SBRT) of non-small cell lung tumor adhered to chest wall or diaphragm
}

\author{
Yi Li, Jing-lu Ma, Xin Chen, Feng-wen Tang and Xiao-zhi Zhang*
}

\begin{abstract}
Background: Large tumor motion often leads to larger treatment volumes, especially the lung tumor located in lower lobe and adhered to chest wall or diaphragm. The purpose of this work is to investigate the impacts of planning target volume (PTV) margin on Stereotactic Body Radiotherapy (SBRT) in non-small cell lung cancer (NSCLC).

Methods: Subjects include 20 patients with the lung tumor located in lower lobe and adhered to chest wall or diaphragm who underwent SBRT. Four-dimensional computed tomography (4DCT) were acquired at simulation to evaluate the tumor intra-fractional centroid and boundary changes, and Cone-beam Computer Tomography (CBCT) were acquired during each treatment to evaluate the tumor inter-fractional set-up displacement. The margin to compensate for tumor variations uncertainties was calculated with various margin calculated recipes published in the exiting literatures.

Results: The means ( \pm standard deviation) of tumor centroid changes were $0.16( \pm 0.13) \mathrm{cm}, 0.22( \pm 0.15) \mathrm{cm}$, and 1.37 $( \pm 0.81) \mathrm{cm}$ in $\mathrm{RL}, \mathrm{AP}$, and $\mathrm{SI}$ directions, respectively. The means ( \pm standard deviation) of tumor edge changes were 0.21 $( \pm 0.18) \mathrm{cm}, 0.50( \pm 0.23) \mathrm{cm}$, and $0.19( \pm 0.44) \mathrm{cm}$ in RL, AP, and SI directions, respectively. The means ( \pm standard deviation) of tumor set-up displacement were $0.03( \pm 0.24) \mathrm{cm}, 0.02( \pm 0.26) \mathrm{cm}$, and $0.02( \pm 0.43) \mathrm{cm}$ in RL, AP, and SI directions, respectively. The PTV margin to compensate for lung cancer tumor variations uncertainties were $0.88,0.98$ and $2.68 \mathrm{~cm}$ in RL, AP and SI directions, which were maximal among all margin recipes.

Conclusions: $4 \mathrm{DCT}$ and $\mathrm{CBCT}$ imaging are appropriate to account for the tumor intra-fractional centroid, boundary variations and inter-fractional set-up displacement. The PTV margin to compensate for lung cancer tumor variations uncertainties can be obtained. Our results show that a conventional $1.0 \mathrm{~cm}$ margin in the SI plane dose not suffice to compensate the geometrical variety of the tumor located in lower lobe and adhered to chest wall and diaphragm.
\end{abstract}

Keywords: 4DCT, CBCT, SBRT, PTV margin, NSCLC

\section{Background}

Lung cancer is one of the leading causes of death in cancer patients in China. According to the literature, 3-year local control rate of the lung cancer is only $66 \%$ in conventional conformal radiotherapy [1]. The stereotactic body radiation therapy (SBRT) is a new technology which can effectively improve the treatment effect of lung cancer. A meta-analysis of 34 lung cancer SBRT studies

\footnotetext{
* Correspondence: zhang9149@sina.com

Department of Radiation Oncology, The First Affiliated Hospital, Xi'an Jiaotong University of Medical College, Xi'an, Shaanxi 710061, China
}

including 2578 patients reported that 3-year local control rate for tumors measuring greater than $3 \mathrm{~cm}$ was $87 \%$ [2]. SBRT delivers high dose in fewer fractions (3-10 fractions) than conventional radiotherapy which may increase toxicity in adjacent organs at risk (OAR). It is therefore critical to minimize the irradiated volume of healthy tissue when delivering the prescribed dose to tumor in SBRT.

However, a variety of geometrical uncertainty such as respiratory motion $[3,4]$, baseline variation $[5-8]$ and setup error [9], limit the precision of radiation therapy (RT) for lung cancer. According to ICRU report 62\#, internal 
margin (IM) and set-up margin (SM) should be included in the PTV to compensate geometrical uncertainties including tumor centroid movement [10], tumor boundary $[11,12]$ and set-up displacement, especially in SBRT. However, the tumor boundary displacement merely discussed about margin calculation previously. It would be assessed in present study.

As a standard modality to compensate tumor motion, four-dimensional CT (4DCT) has been generally applied to the calculation of the extent margin of tumor motion [13], and is also incorporated into treatment planning. When only areas with tumor appearance are included in the target volume definition, there is a significant reduction of the mean volume by the use of 4DCT [14]. Generally, the need of contour gross tumor volume (GTV) in up to ten phases of respiration is a time-consuming procedure for the routine clinical use of 4DCT scans. To reduce the workload of multiple contour delineations in 4DCT, maximum intensity projection (MIP) and averaged intensity (AI) of 4DCT image are reconstructed in order to be used in the treatment planning, MIP provide a 3D CT scan whose vowels' values are the greatest vowels' intensity values throughout the 4D CT dataset. AI provides a 3D CT scan whose vowels' values are the arithmetic mean of the 4D CT scans [15]. However, the tumor contoured with the MIP or AI series cannot totally represent the true tumor size, location and shape, especially adhered to chest wall or diaphragm [15]. To correctly assess the tumor motion and tumor shape, ten phase of the 4DCT were contoured in our study.

Tumor localization is also a significant issue in NSCLC SBRT, the major focus has been the development of image-guidance system. Several image guidance techniques such as electronic portal imaging device (EPID) [16] and CBCT [17], have been adapted to narrow the setup margin. Among them, three-dimensional (3D) matching, coupled with $\mathrm{CBCT}$ provides results that have greater accuracy than two-dimensional matching, coupled with EPID [18]. Generally, with the repeating CBCT imaging, intra-fractional and inter-fractional set-up displacement can be assessed for the lung cancer patients' set-up displacement using KV X-ray,such as fluoroscopy, CBCT and four dimensional CBCT (4D-CBCT) [19-21]. CBCT imaging is sufficient to account for the inter-fractional tumor variation and setup variation based on the bone structure, which was adopted in our study. Furthermore, size, patients characteristics are also significant factors to influent patients' set-up margin [22], but these factors are negligible in the set-up margin.

The present study would evaluate changes in tumor motion magnitude and set-up error by 4DCT at planning and $\mathrm{CBCT}$ at treatment, and calculated the clinical target volume (CTV)-planning target volume (PTV) margins to compensate for these changes, which may minimize the risk of complications and tumor failures. The aim of the present study is to decide whether conventional $1.0 \mathrm{~cm}$ margin in the SI plane and $0.5 \mathrm{~cm}$ margin in the other plane suffice to compensate the geometrical variety of the tumor adhere to chest wall and diaphragm.

\section{Methods}

\section{Patient and tumor characteristics}

Between February 2013 and June 2015, 20 patients were recruited to this study to assess PTV margin. Table 1 summarizes the patient and tumor's general information and detailed characteristics.

The studies were approved by the Institutional Committee for Clinical Research and the Local Ethics Committee in The First Affiliated Hospital, Xi'an Jiaotong University of medical college. Eligible patients were given written informed consent.

\section{DCT simulation and image acquisition}

During the CT simulation, all patients were immobilized using thermoplastic mask in the supine position with arms raised above the head. Then, each patient received a helical treatment planning three-dimensional computed tomography (TP-3DCT) and an additional 4DCT simulation under free breathing condition on a 16-slice CT scanner (Big bore, Philips Medical Systems, Cleveland, OH). For TP-3DCT, each scan's rotation time took about $1 \mathrm{~s}$ to acquire image dates. The other parameters were as follows: pitch $0.85,120 \mathrm{KV}$ and $400 \mathrm{mAs}$. During $4 \mathrm{DCT}$ scanning, the respiratory signal was acquired by a pressure sensor ("pneumonia bellows"). The pressure in the bellows decreases as the patient inhales, with the

Table 1 Patients and tumor generally characteristics of 20 patients in this study

\begin{tabular}{ll}
\hline & Number \\
\hline Sex & 12 \\
Male & 8 \\
Female & \\
Age (years) & 7 \\
$>60$ & 13 \\
$\leq 60$ & \\
Tumor volume $\left(\mathrm{cm}^{3}\right)$ & 9 \\
$>40$ & 11 \\
$\leq 40$ & \\
Side & 6 \\
Right & 14 \\
Left & \\
Site & 15 \\
Chest wall adherent & 5 \\
Diaphragm adherent &
\end{tabular}


opposite happening as the patient exhales. Then, the pressure change was measured by a remote pressure sensor which provided a direct-current voltage to an analog-to-digital converter. 4DCT images were reconstructed and then sorted into 10 respiratory phases, with $0 \%$ representing end-inhalation and 50\% representing end-exhalation. Prospectively phase-binned image sets was been used to extract displacement binned 4DCT. Both the TP-3DCT and 4DCT images were reconstructed with a thickness of $3 \mathrm{~mm}$ and then transferred to a Pinnacle V. 9.0y treatment planning system (Philips Medical Systems, Fitchburg, USA).

\section{Manual contouring and treatment planning}

For target volume definition, the 4DCT phases display the extreme of tumor positions for each spatial direction. After the matching of CT-cubes between 4DCT phases and 3DCT phase in the Philips treatment planning system, GTV contours were manually delineated on the 10 phases of the 4DCT scan using the lung window setting by the same physician to make the inter-observer lowest as soon as possible $(\mathrm{L}=-600 \mathrm{Hu}, \mathrm{W}=1600 \mathrm{Hu}$ [20]), and $\mathrm{CTV}_{4 \mathrm{D}}$ was defined as $\mathrm{GTV}_{4 \mathrm{D}}$ plus an isotropic margin of $0.6 \mathrm{~cm}$ for adenoid carcinoma and the one of $0.8 \mathrm{~cm}$ for squamous carcinoma. The combined volume of each CTVs in 10 phases was defined as internal $\mathrm{CTV}_{4 \mathrm{D}}$ (ITV). The modification to form the PTV $\mathrm{PD}_{4 \mathrm{D}}$ was performed by adding an isotropic margin of $3 \mathrm{~mm}$ to ITV to account for set-up inaccuracies in all direction (Fig. 1). The $\mathrm{PTV}_{4 \mathrm{D}}$ contours on 4DCT phases were projected to the contour named PTV on the TP-3DCT.

For treatment planning, every patient received an individual treatment plan and the plan calculation were performed on 3DCT, 10 patients were treated with the IMRT technology, and the others with conformal arc technology. All patients were prescribed total doses of $48 \mathrm{~Gy}$ in 4 fractions $[16,23,24]$ with the $95 \%$ isodose covering the PTV.

\section{Tumor boundary change definition}

Figure 2 shows tumor boundary change definition. Ten beams with gantry angle $0^{\circ}$ and $90^{\circ}$ were used and conformed to GTV of each phase with centroid of corresponding GTV for each patient. The values of jaw location in the RL, and SI directions with beam of angle $0^{\circ}$ were collected. Then the same values with beam of angle $90^{\circ}$ were also collected. The variation of these values represent the boundary displacement in the RL, AP and SI direction.

\section{CBCT simulation and image acquisition}

The on-board imager integrated in a Synergy medical linear accelerator (Elekta, Stockholm, Sweden) was adopted to acquire $\mathrm{CBCT}$ images. Before acquiring the image data, each patient was immobilized in the same position as that used in 4DCT scan. A $120 \mathrm{kV}$ x-ray tube voltage and $400 \mathrm{mAs}$ current were used for generating middleresolution images. The scan angle was from $-178^{\circ}$ to $178^{\circ}$. All CBCT images were reconstructed with a thickness of $3 \mathrm{~mm}$. After each patient were initial positioned, a CBCT was acquired and registered to the planning TP-3DCT based on the location of vertebral bodies (bony landmarks) (Fig. 3). The target position error was corrected by shifting the treatment couch. If the position error was $\leq 2 \mathrm{~mm}$ in all three direction, the patient was treated without shifting couch; else couch was shifted and a second $\mathrm{CBCT}$ was acquired to measure the residual error until a residual error was $\leq 2 \mathrm{~mm}$ [19]. At the end of treatment, a $\mathrm{CBCT}$ scan was finally performed to assess the intrafractional set-up displacement.

\section{Respiration motion analysis}

Centroid position and boundary of the respective $\mathrm{CTV}_{4 \mathrm{D}}$ were calculated and compared. The centroid shift of respective $\mathrm{CTV}_{4 \mathrm{D}}$ was represented as tumor position variation, and the tumor boundary change of respective $\mathrm{CTV}_{4 \mathrm{D}}$ was represented as tumor shape variation during respiration. The 3D spatial motion vector of the individually $\mathrm{CTV}_{4 \mathrm{D}}$ centroid and boundary variation were evaluated according to the recipe as follows [25]

$$
V=\sqrt{R L^{2}+A P^{2}+S I^{2}}
$$

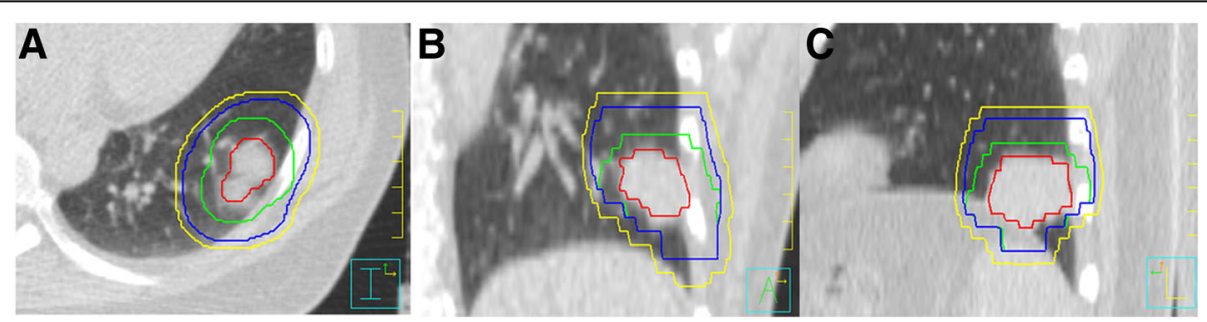

Fig. 1 Phase 0\% of four-dimensional computed tomography (4DCT) image for an protocol NSCLC patient. (a) transverse (b) sagittal (c) coronal; Gross tumor volume (GTV) of phase $0 \%$ in Red; Clinical target volume (CTV) of phase $0 \%$ in Green; Internal clinical target volume (ITV) in blue; Planning target volume (PTV) in Yellow 


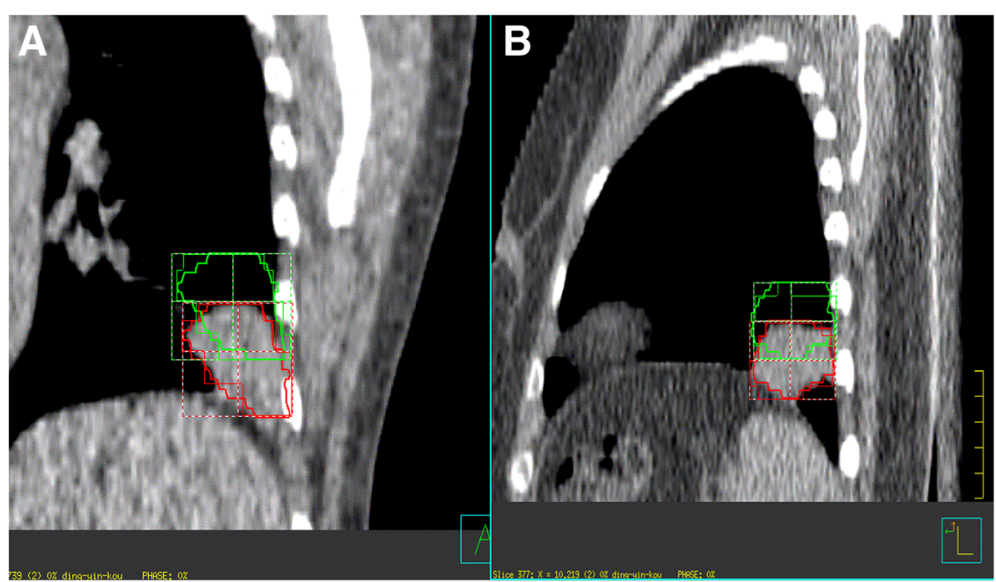

Fig. 2 Phase 0\% of four-dimensional computed tomography (4DCT) image for an protocol NSCLC patient. (a) coronal (b) sagittal; Gross tumor volume (GTV) of phase $0 \%\left(\mathrm{GTV}_{0 \%}\right)$ in Red; Gross target volume (GTV) of phase $50 \%\left(\mathrm{GTV}_{50 \%}\right)$ in Green

\section{Margin calculation}

Existing literature has proposed approaches to calculate the PTV margin based on systematic and random uncertainties (see Table 2) [8, 26-28], which were applied for the PTV margin calculation in our study. Among these approaches, the recipes approached by Stroom and van Herk are widely adopted in current clinical treatment. In all recipes, $\Sigma$ represents the standard deviation (SD) of systematic errors and $\sigma$ is the root-mean square of random errors. In this study, the systematic errors include the standard deviat55ion (SD) of the centroid movement denoted as $\Sigma_{\text {centroid }}$, the tumor boundary changes denoted as $\Sigma_{\text {boundary }}$ and set-up displacement denoted as $\sum_{\text {setup }}$ of each patient. As each patient had one 4DCT scan, the random errors of the centroid and boundary changes were zero, and the total random errors equal to set-up random errors. As a result, the total SD of systematic errors $\sum_{\text {total }}$ and random errors were evaluated with the recipe: $\sum_{\text {total }}$ $=\sqrt{\sum_{\text {centroid }}^{2}+\sum_{\text {boundary }}^{2}+\sum_{\text {setup }}^{2}}, \sigma_{\text {total }}=\sigma_{\text {setup }}$.

\section{Data analysis}

SPSS 19.0 was used for statistical analysis. Differences were considered significant for $P<0.05$. For test of correlation, it is Person's rank test which was used.

\section{Results}

Tumor centroid and boundary changes

Figures 4 and 5 show the tumor boundary and centroid changes for 20 patients. A positive value in the right-left (RL), anterior-posterior (AP), and superiorinferior (SI) directions represents a shift in the right, anterior, and inferior directions, respectively. We did not find any statistically significant correlation between tumor centroid displacement or boundary changes and patients' age. The mean 3D centroid change value was $1.55 \pm 0.46 \mathrm{~cm}$, with a maximum at $2.33 \mathrm{~cm}$. Tumor centroid had a greater mean changes in SI direction than in other direction (SI vs RL: $\mathrm{t}=$ $0.587, \mathrm{P}<0.05$; SI vs AP: $\mathrm{t}=0.547, \mathrm{P}<0.05$ ). The mean $3 \mathrm{D}$ boundary change value was $0.70 \pm 0.33 \mathrm{~cm}$, with a

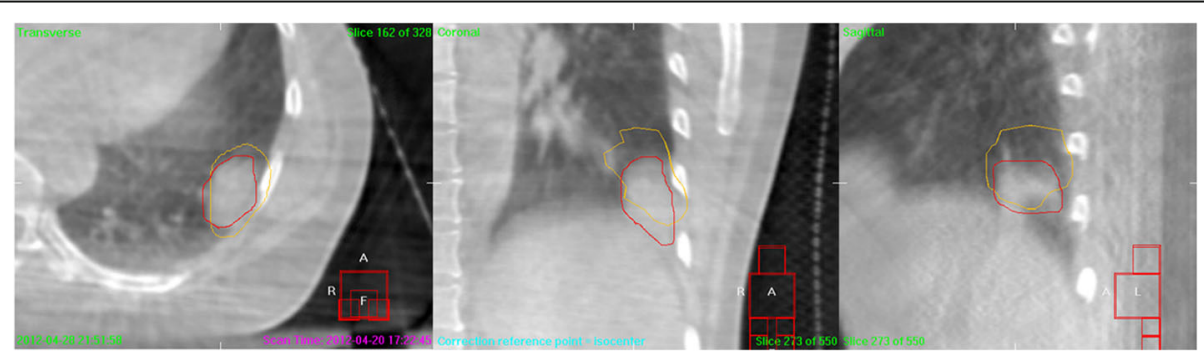

Fig. 3 Precorrection cone-beam CT (CBCT) image for an protocol NSCLC patient in the thermoplastic mask. TP-3DCT GTV in Yellow; Precorrection GTV in Red 
Table 2 Summary of various published recommendations for margins around target volumes (CTV)

\begin{tabular}{lll}
\hline Author & Region & Recipe \\
\hline Stroom et al.(1999a) [26] & PTV & $2 \sum+0.7 \sigma$ \\
Van Herk et al.(2000) [27] & PTV & $2.5 \Sigma+0.7 \sigma$ \\
Parker et al. (2002) [28] & PTV & $\sum+\sqrt{\left(\sigma^{2}+\sum^{2}\right)}$ \\
Snoke JJ (2007) & PTV & $2.5 \Sigma+\beta\left(\sigma^{2}+\sigma_{P}^{2}\right)^{1 / 2}-\beta \sigma_{P}$
\end{tabular}

Symbols: $\Sigma$, standard deviation of systematic uncertainties; $\sigma$, standard deviation of statistical (random) uncertainties. $\sigma_{P}=0.64, \beta=0.84$.

maximum at $1.40 \mathrm{~cm}$. Tumor boundary motion had a greater mean displacement in AP direction than in other directions, but it is not statistically significant, which is similar to the one observed by Gauthier et al. [12]. In all direction, the average boundary changes directions were toward to the centroid changes directions, which means the tumor shrank as expected.

\section{Tumor set-up errors}

Figure 6 shows tumor set-up errors in RL, AP, and SI directions for 80 pre-correction CBCT fractions. A positive value in the right-left (RL), anteriorposterior (AP), and superior-inferior (SI) directions represents a shift in the right, anterior, and inferior directions, respectively. The maximum set-up error were as large as $0.66 \mathrm{~cm}$ in RL direction, $0.54 \mathrm{~cm}$ in $\mathrm{AP}$ direction, and $1.16 \mathrm{~cm}$ in SI direction. The average setup errors are $0.13 \pm 3.15 \mathrm{~cm}, 0.37 \pm 2.76 \mathrm{~cm}$ and $0.20 \pm 5.73 \mathrm{~cm}$ in RL, AP and SI directions, respectively. From the distribution, we could calculated the probability of having set-up errors lager than $5 \mathrm{~mm}$ (which is the margin used for set-up margin) and found it to be $15.0 \%$ in $\mathrm{RL}, 6.3 \%$ in $\mathrm{AP}$, and $38.8 \%$ in SI directions, respectively. The mean set-up errors had a greater value in SI direction than in

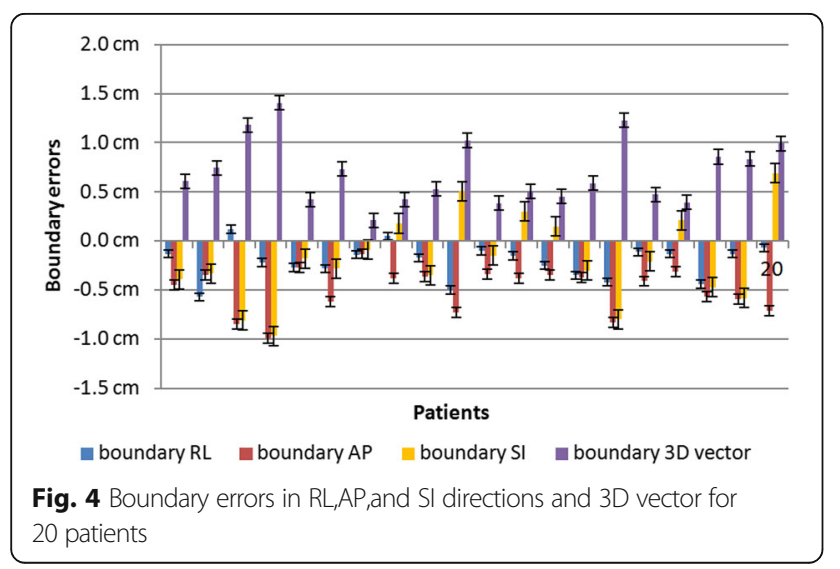

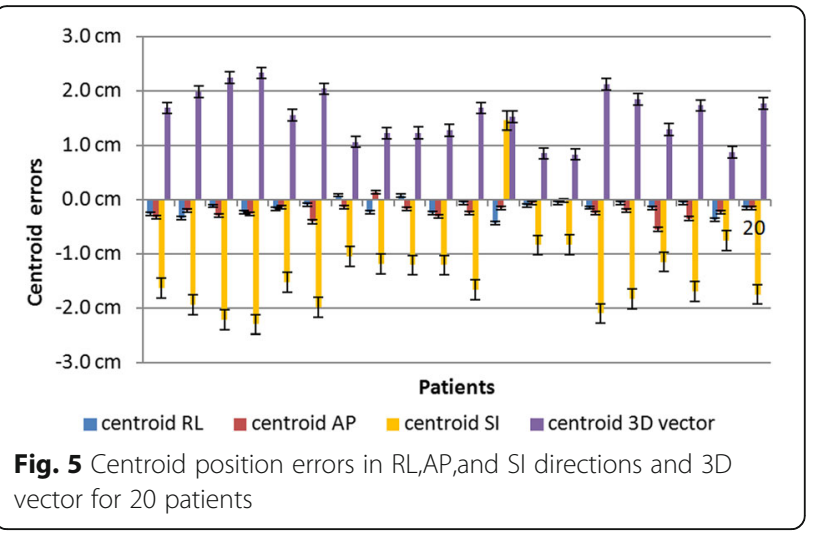

other directions. (SI vs AP: $\mathrm{r}=-0.609, \mathrm{p}<0.05$; SI vs $R L: r=-0.480, p>0.05)$.

\section{PTV margin}

Table 3 shows the SDs of systematic $(\Sigma)$ and random $(\sigma)$ errors. One patient had one 4DCT scan at tumor CT simulation, and therefore the random errors were zero for tumor centroid and boundary displacement. Based on the SDs of set-up systematic and random errors in previous table, the pre-correction set-up margin was calculated and showed in Table 4 . Among all recipes, the maximum were 0.67, 0.69 and $1.21 \mathrm{~cm}$ in RL, AP and SI directions, which were calculated with the recipe by van Herk et al. To compensate all geometrical uncertain motioned above, the total margin were calculated and showed in Table 4. Among all recipes, the maximum were $0.88,0.98$ and $2.68 \mathrm{~cm}$ in RL, AP and SI directions, which were also calculated with the recipe by van Herk et al.

\section{Discussion}

It is reported [29] that the tumor movement in lung is not related to patient height, weight, cancer stage and lung function, and therefore these issues were not included in this study. We evaluated changes in tumor motion magnitude and set-up error by 4DCT scan at planning and CBCT scan at treatment and calculated the margins to compensate for these changes $[16,17]$. To our knowledge, this is the first study to report the evaluation of tumor centroid, boundary changes and set-up displacement, which are account into the margins calculation.

We admit that at the time we performed this study, we did not perform Post-correction and post-treatment CBCT to verify that set-up displacement and tumor locatioin had not changed. However, [30,31] existing literatures have shown that post-correction margins based on bony structure are negligible to account into PTV margin, which are less than $2 \mathrm{~mm}$ in three directions [24]. Other literature has shown that inter-fractional tumor position and breathing motion based on bony 


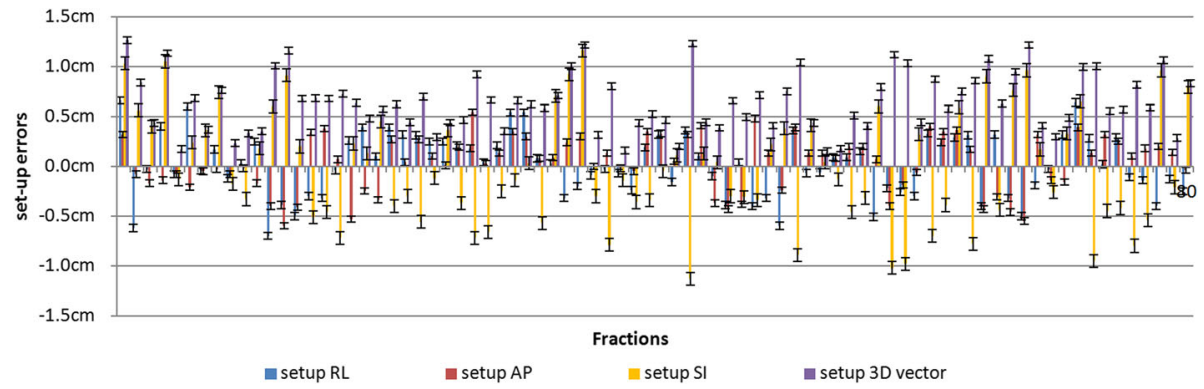

Fig. 6 Set-up errors in in RL, AP, and SI directions and 3D vector for 80 fractions. Data were derived based on bony landmark alignments

structure during treatment are stable. Therefore, the post-treatment margins are also negligible to account into PTV margin. In addition, we did perform postcorrection and post-treatment CBCT on other patients and found that the tumor with post-correction and posttreatment displacement changed is negligible, and therefore the data have not yet been published.

The variation in the centroid changes between ITV based from 4DCT and CTV based from 3DCT was not significant [32]. Therefore, the intra-fractional tumor motion on 4DCT different phase can represent the one on the TP-3DCT. Intra-fractional tumor motion in present study was divided into tumor centroid and boundary displacement under free-breathing conditions. Centroid and boundary variation were collected starting from $0 \%$ phase to ending at a phase of maximum variation, which was $50 \%$ phase mostly. So the variation of centroid and boundary were collected starting from inferior direction to ending at superior direction. In my study, A positive value in superiorinferior (SI) directions represents a shift in the inferior directions. So the values for centroid an boundary in the graphs are negative values. Mori, S et al. [33] quantified the magnitude of intra-fraction lung tumor motion under free-breathing conditions using 4DCT and reported the mean ( \pm standard deviation) tumor centroid motion were $0.19( \pm 0.16), 0.40( \pm 0.23)$, and $1.03( \pm 0.71) \mathrm{mm}$ in the RL, AP, and SI directions respectively, which was similar with the present study result. Only the motion in SI direction was less than

Table 3 The Standard deviation of systematic $(\Sigma)$ and random $(\sigma)$ errors

\begin{tabular}{llllllll}
\hline & $\Sigma$ & & & & $\sigma$ & & \\
\cline { 2 - 3 } & $\mathrm{RL}$ & $\mathrm{AP}$ & $\mathrm{SI}$ & & $\mathrm{RL}$ & $\mathrm{AP}$ & $\mathrm{SI}$ \\
\hline Centroid point & 0.14 & 0.18 & 0.55 & & & \\
Boundary & 0.19 & 0.21 & 0.17 & & & \\
Set-up & 0.24 & 0.26 & 0.43 & 0.10 & 0.08 & 0.20 \\
\hline
\end{tabular}

Symbols: $\sum$, standard deviation of systematic uncertainties; $\sigma$, standard deviation of statistical (random) uncertainties present study. We presume that only a few lung tumors were located in the lower lobe on previous study, resulting in their small centroid changes in SI direction.

Tumor boundary motion, which was merely discussed on previous PTV margin study, were quantified in present study and found that the maximum were $0.57,0.99$, and $0.97 \mathrm{~cm}$ in RL, AP and SI direction respectively. We could calculated the probability of having boundary motion lager than $5 \mathrm{~mm}$ and found it to be $10.0 \%$ in $\mathrm{RL}, 40.0 \%$ in $\mathrm{AP}$, and $7.5 \%$ in SI directions, respectively. We presume that the tumor located in lower lobe patients was adhered to the diaphragm, resulting in their significant boundary changes. We believe that the boundary changes in intra-fractional variation is significant, especially in SBRT treatment.

Set-up error in the present study was defined as the shift from TP-3DCT to CBCT. This error includes the inter-fractional set-up error based on bony structure. The standard deviation of set-up systematic errors $(\Sigma)$ are $0.24,0.26$ and $0.43 \mathrm{~cm}$ in RL, AP and SI directions. The result was similar with the one observed by Worm, E.S. et al., which was $0.23,0.16$ and $0.45 \mathrm{~cm}$ [31]. The standard deviation of set-up random errors $(\Sigma)$ are 0.10 , 0.08 and $0.20 \mathrm{~cm}$ in RL, AP and SI directions. The result was also similar with the one observed by $\mathrm{Li}, \mathrm{W}$ ea al, which was $0.12,0.13$ and $0.17 \mathrm{~cm}$ with Performance Status 0 patients stratified by Eastern Cooperative Oncology Group performance [24]. The average setup errors are $0.13 \pm 3.15 \mathrm{~cm}, 0.37 \pm 2.76 \mathrm{~cm}$ and $0.20 \pm 5.73 \mathrm{~cm}$ in $\mathrm{RL}$, $\mathrm{AP}$ and SI directions, respectively. And the maximum set-up margin in the present study was 0.67, 0.69 and $1.21 \mathrm{~cm}$ in RL, AP and SI directions calculated with van Herk recipe. These result was similar with the study by Wang L et al. [23], which assessed set-up margin using three-dimensional CBCT and report it to be $0.9-1.0 \mathrm{~cm}$ range. However, the study by Ueda $\mathrm{Y}$ et al. [16] assessed set-up margin by shift from MTP-4DCT at planning to MTP-cine at the treatment and reported it to be $0.52 \mathrm{~cm}$ in SI direction in $98 \%$ session's calculated 
Table 4 CTV margin changes of NSCLC patients

\begin{tabular}{|c|c|c|c|c|c|c|c|}
\hline \multirow[t]{2}{*}{ Author } & \multirow[t]{2}{*}{ Recipe } & \multicolumn{3}{|c|}{ Margin through set-up errors } & \multicolumn{3}{|c|}{ Margin through all variation } \\
\hline & & $\mathrm{ML}$ & $A P$ & $\mathrm{SI}$ & $\mathrm{ML}$ & AP & $\mathrm{SI}$ \\
\hline $\begin{array}{l}\text { Stroom et al. } \\
\text { (1999a) [26] }\end{array}$ & $2 \Sigma+0.7 \sigma$ & 0.55 & 0.57 & 0.99 & 0.72 & 0.79 & 2.18 \\
\hline $\begin{array}{l}\text { van Herk et al. } \\
\text { (2000) [27] }\end{array}$ & $2.5 \Sigma+0.7 \sigma$ & 0.67 & 0.69 & 1.21 & 0.88 & 0.98 & 2.68 \\
\hline $\begin{array}{l}\text { Parker et al. } \\
\text { (2002) [28] }\end{array}$ & $\sum+\sqrt{\left(\sigma^{2}+\sum^{2}\right)}$ & 0.50 & 0.52 & 0.90 & 0.66 & 0.75 & 2.06 \\
\hline $\begin{array}{l}\text { Snoke JJ } \\
(2007)\end{array}$ & $2.5 \Sigma+\beta\left(\sigma^{2}+\sigma_{P}^{2}\right)^{1 / 2}-\beta \sigma_{P}$ & 0.60 & 0.64 & 1.09 & 0.82 & 0.93 & 2.57 \\
\hline
\end{tabular}

Stroom recipe (Table 2). The margin in study above is less than that in present study. We presume that their set-up, which used Body Fix double-vacuum system, resulted in their smaller set-up error.

The PTV margin also depends on the patient positioning techniques. Corradetti MN et al. [34] Stated that margins were $0.49 \mathrm{~cm}, 0.85 \mathrm{~cm}$ and $0.77 \mathrm{~cm}$ in RL, AP, and SI direction calculated by the van Herk recipe, respectively, which is smaller than the present result. We presume that their set-up, which used the full-body vacuum cushion system, resulted in their smaller inter-fractional error. Many other studies on SBRT have adopted $5 \mathrm{~mm}$ as the PTV margin using various advanced immobilized devices, such as Microtron, BodyFix. These devices can obviously reduce the PTV margin in the SBRT treatment for NSCLC. However, these advanced devices cause more time in the patients' set-up procedure and bring more variations in the time spent on the treatment couch [17].

A total PTV margin combining tumor centroid, boundary changes and set-up displacement were $0.88,0.98$ and $2.68 \mathrm{~cm}$ in RL, AP and SI directions, respectively, which were maximum among all margin recipes. According to the comments of Table 5, the statistical assumption with van Herk's recipe is minimum absorbed dose to CTV is $95 \%$ for $90 \%$ of patients. It's requirement about minimum absorbed dose is high in realistic treatment plans, so the bigger margin size are needed to meet the requirement. This appoint is proved in my study, which shows that among all recipes, the maximum margin were calculated with the recipe by van Herk et al. And the requirement of
Parker's recipe about minimum absorbed dose is the same as one of van Herk's, but the probability lever is not specified. And the results in my study shows that the margin calculated with Parker's recipe is less than one with van Herk's recipe.

Many studies stated that PTV margin was $0.5 \mathrm{~cm}$ in the axial plane and $1.0 \mathrm{~cm}$ in the cranial-caudal plane in all directions in the study on SBRT for NSCLC [20, 35-37]. The results were less than the one observed in SI direction of the present study. In conclusion, a $0.5 \mathrm{~cm}$ margin in the axial plane suffices to compensate the geometrical variety; nevertheless, a $1.0 \mathrm{~cm}$ margin in the SI plane is dose not compensated the geometrical variety in the present study, which the tumor located in lower lobe is adhered to chest wall and diaphragm.

\section{Conclusions}

4DCT imaging is appropriate to account for the intrafractional tumor centroid position, and boundary variations, and CBCT imaging is appropriate to account for the inter-fractional tumor set-up variation. We observed that the large tumor geometrical motion in lung tumor located in lower lobe and adhered to chest wall and diaphragm on SBRT for NSCLC and PTV margin could be as large as $0.88,0.98$ and $2.68 \mathrm{~cm}$ in RL, AP and SI directions, respectively. However, a conventional $1.0 \mathrm{~cm}$ margin in the SI plane dose not suffice to compensate the geometrical variety of the tumor adhere to chest wall and diaphragm.

Table 5 Summary of various published recommendations for margins around target volumes

\begin{tabular}{|c|c|c|}
\hline Author & Recipe & Comments \\
\hline Stroom et al. (1999a) [26] & $2 \Sigma+0.7 \sigma$ & 95\% absorbed dose to on average $99 \%$ of CTV tested in realistic plans. \\
\hline Van Herk et al. (2000) [27] & $2.5 \Sigma+0.7 \sigma$ & $\begin{array}{l}\text { Minimum absorbed dose to CTV is } 95 \% \text { for } 90 \% \text { of patients. Analytical solution for perfect } \\
\text { conformation. }\end{array}$ \\
\hline Parker et al. (2002) [28] & $\sum+\sqrt{\left(\sigma^{2}+\sum^{2}\right)}$ & $\begin{array}{l}95 \% \text { minimum absorbed dose and } 100 \% \text { absorbed dose for } 95 \% \text { of volume. Probability levels not } \\
\text { specified. }\end{array}$ \\
\hline Snoke JJ (2007) & $\begin{array}{l}2.5 \sum+\beta\left(\sigma^{2}+\sigma_{p}^{2}\right)^{1 / 2} \\
-\beta \sigma_{p}\end{array}$ & Minimum $95 \%$ of the prescribed dose for $90 \%$ of patients \\
\hline
\end{tabular}




\section{Abbreviations}

4DCT: Four-dimensional computed tomography; CBCT: Cone-beam Computer Tomography; CTV: Clinical target volume; EPID: Electronic portal imaging device; GTV: Gross target volume; ITV: Internal clinical target volume; NSCLC: Non-small cell lung cancer; PTV: Planning target volume; SBRT: Stereotactic Body Radiotherapy; TP-3DCT: Treatment planning threedimensional computed tomography

\section{Acknowledgments}

All authors in this study and many colleagues helped to collect and analyze data. Their support and helps should be appreciated.

\section{Funding}

This study was supported in part by institutional science foundation of the first affiliated hospital of Xi'an Jioatong University (2013YK27).

\section{Availability of data and materials}

All data and materials have been presented in the manuscript.

\section{Authors' contributions}

X-ZZ conceived of the study, and participated in its design and coordination. $Y L$ design and carried out the studies, participated in the sequence alignment and drafted the manuscript. J-LM help to draft the manuscript and contributed to the writing of the manuscript, XC and F-WT help to carried out the studies. All authors read and approved the final manuscript.

\section{Competing interests}

The authors declare that they have no competing interests.

\section{Consent for publication}

Not applicable.

\section{Ethics approval and consent to participate}

The study was approved by the Institutional Committee for Clinical Research and the Local Ethics Committee in The First Affiliated Hospital, Xi'an Jiaotong University of medical college. Eligible patients were given written informed consent.

Received: 24 April 2016 Accepted: 31 October 2016

\section{Published online: 15 November 2016}

\section{References}

1. Lanni Jr TB, Grills IS, Kestin LL, Robertson JM. Stereotactic radiotherapy reduces treatment cost while improving overall survival and local control over standard fractionated radiation therapy for medically inoperable nonsmall-cell lung cancer. Am J Clin Oncol. 2011;34(5):494-8. doi:10.1097/COC Ob013e3181ec63ae. Epub 2010/09/02

2. Curran Jr WJ, Paulus R, Langer CJ, Komaki R, Lee JS, Hauser S, et al. Sequential vs. concurrent chemoradiation for stage III non-small cell lung cancer: randomized phase III trial RTOG 9410. J Natl Cancer Inst. 2011; 103(19):1452-60. doi:10.1093/jnci/djr325. PubMed PMID: 21903745, PubMed Central PMCID: PMC3186782, Epub 2011/09/10.

3. Borst GR, Sonke JJ, Betgen A, Remeijer P, van Herk M, Lebesque JV. Kilovoltage cone-beam computed tomography setup measurements for lung cancer patients; first clinical results and comparison with electronic portalimaging device. Int J Radiat Oncol Biol Phys. 2007;68(2):555-61. doi:10.1016/ j.jijobp.2007.01.014. Epub 2007/04/03.

4. de Boer HC, van Sornsen de Koste JR, Senan S, Visser AG, Heijmen BJ. Analysis and reduction of $3 \mathrm{D}$ systematic and random setup errors during the simulation and treatment of lung cancer patients with CT-based external beam radiotherapy dose planning. Int J Radiat Oncol Biol Phys. 2001:49(3):857-68. Epub 2001/02/15

5. Abbas G, Pennathur A, Landreneau RJ, Luketich JD. Radiofrequency and microwave ablation of lung tumors. J Surg Oncol. 2009;100(8):645-50. doi: 10.1002/jso.21334. Epub 2009/12/18.

6. Chang J, Mageras GS, Yorke E, De Arruda F, Sillanpaa J, Rosenzweig KE, et al. Observation of interfractional variations in lung tumor position using respiratory gated and ungated megavoltage cone-beam computed tomography. Int J Radiat Oncol Biol Phys. 2007;67(5):1548-58. doi:10.1016/j. ijrobp.2006.11.055. PubMed PMID: 17394950, PubMed Central PMCID: PMC2278042, Epub 2007/03/31
7. Hugo GD, Yan D, Liang J. Population and patient-specific target margins for $4 \mathrm{D}$ adaptive radiotherapy to account for intra- and inter-fraction variation in lung tumour position. Phys Med Biol. 2007;52(1):257-74. doi:10.1088/00319155/52/1/017. Epub 2006/12/22.

8. Sonke JJ, Lebesque J, van Herk M. Variability of four-dimensional computed tomography patient models. Int J Radiat Oncol Biol Phys. 2008;70(2):590-8. doi:10.1016/j.jirobp.2007.08.067. Epub 2007/11/27.

9. Seppenwoolde Y, Shirato H, Kitamura K, Shimizu S, van Herk M, Lebesque $J \mathrm{~V}$, et al. Precise and real-time measurement of 3D tumor motion in lung due to breathing and heartbeat, measured during radiotherapy. Int I Radiat Oncol Biol Phys. 2002:53(4):822-34. Epub 2002/07/04.

10. Britton KR, Starkschall G, Tucker SL, Pan T, Nelson C, Chang JY, et al. Assessment of gross tumor volume regression and motion changes during radiotherapy for non-small-cell lung cancer as measured by fourdimensional computed tomography. Int J Radiat Oncol Biol Phys. 2007; 68(4):1036-46. doi:10.1016/j.jjobp.2007.01.021. Epub 2007/03/24.

11. Donnelly ED, Parikh PJ, Lu W, Zhao T, Lechleiter K, Nystrom M, et al. Assessment of intrafraction mediastinal and hilar lymph node movement and comparison to lung tumor motion using four-dimensional CT. Int J Radiat Oncol Biol Phys. 2007;69(2):580-8

12. Gauthier JF, Varfalvy N, Tremblay D, Cyr MF, Archambault L. Characterization of lung tumors motion baseline using cone-beam computed tomography. Med Phys. 2012;39(11):7062-70. doi:10.1118/1.4762563. Epub 2012/11/07.

13. Ecclestone $G$, Bissonnette JP, Heath E. Experimental validation of the van Herk margin formula for lung radiation therapy. Med Phys. 2013;40(11): 111721. doi:10.1118/1.4824927. Epub 2013/12/11.

14. Hof H, Rhein B, Haering P, Kopp-Schneider A, Debus J, Herfarth K. 4D-CT-based target volume definition in stereotactic radiotherapy of lung tumours: comparison with a conventional technique using individual margins. Radiother Oncol. 2009:93(3):419-23. doi:10.1016/.jradonc.2009.08.040. Epub 2009/09/29.

15. Bradley JD, Nofal AN, El Naqa IM, Lu W, Liu J, Hubenschmidt J, et al. Comparison of helical, maximum intensity projection (MIP), and averaged intensity (AI) 4D CT imaging for stereotactic body radiation therapy (SBRT) planning in lung cancer. Radiother Oncol. 2006;81(3):264-8. doi:10.1016/j. radonc.2006.10.009. Epub 2006/11/23

16. Ueda Y, Miyazaki M, Nishiyama K, Suzuki O, Tsujii K, Miyagi K. Craniocaudal safety margin calculation based on interfractional changes in tumor motion in lung SBRT assessed with an EPID in cine mode. Int J Radiat Oncol Biol Phys. 2012;83(3):1064-9. doi:10.1016/j.jprobp.2011.07.043. Epub 2012/01/17.

17. Bissonnette JP, Franks KN, Purdie TG, Moseley DJ, Sonke JJ, Jaffray DA, et al. Quantifying interfraction and intrafraction tumor motion in lung stereotactic body radiotherapy using respiration-correlated cone beam computed tomography. Int J Radiat Oncol Biol Phys. 2009;75(3):688-95. doi:10.1016/j. ijrobp.2008.11.066. Epub 2009/04/28.

18. Hugo GD, Liang J, Campbell J, Yan D. On-line target position localization in the presence of respiration: a comparison of two methods. Int J Radiat Oncol Biol Phys. 2007:69(5):1634-41. doi:10.1016/j.jirobp.2007.08.023. PubMed PMID: 18029112, PubMed Central PMCID: PMC2170894, Epub 2007/11/22.

19. Grills IS, Hugo G, Kestin LL, Galerani AP, Chao KK, Wloch J, et al. Imageguided radiotherapy via daily online cone-beam $C T$ substantially reduces margin requirements for stereotactic lung radiotherapy. Int J Radiat Oncol Biol Phys. 2008;70(4):1045-56.

20. Corradetti MN, Mitra N, Bonner Millar LP, Byun J, Wan F, Apisarnthanarax S, et al. A moving target: image guidance for stereotactic body radiation therapy for early-stage non-small cell lung cancer. Practical Radiation Oncology. 2013;3(4):307-15. doi:10.1016/j.prro.2012.10.005. Epub 2014/03/29.

21. Cai W, Dhou S, Cifter F, Myronakis M, Hurwitz MH, Williams CL, et al. $4 \mathrm{D}$ cone beam CT-based dose assessment for SBRT lung cancer treatment. Phys Med Biol. 2016;61(2):554-68. doi:10.1088/0031-9155/61/2/554. Epub 2015/12/20.

22. Liu HW, Khan R, Nugent Z, Krobutschek K, Dunscombe P, Lau H. Factors influencing intrafractional target shifts in lung stereotactic body radiation therapy. Practical Radiation Oncology. 2014;4(1):e45-51. doi:10.1016/j.prro. 2013.02.013. Epub 2014/03/14.

23. Wang L, Feigenberg S, Fan J, Jin L, Turaka A, Chen L, et al. Target repositional accuracy and PTV margin verification using three-dimensional cone-beam computed tomography (CBCT) in stereotactic body radiotherapy (SBRT) of lung cancers. J Appl Clin Med Phys. 2012;13(2):3708. doi:10.1120/jacmp.v13i2.3708. Epub 2012/03/10.

24. Li W, Purdie TG, Taremi M, Fung S, Brade A, Cho BC, et al. Effect of immobilization and performance status on intrafraction motion for stereotactic lung radiotherapy: analysis of 133 patients. Int J Radiat Oncol 
Biol Phys. 2011;81(5):1568-75. doi:10.1016/j.jprobp.2010.09.035. Epub 2010/ $11 / 16$.

25. Rietzel E, Chen GT, Choi NC, Willet CG. Four-dimensional image-based treatment planning: target volume segmentation and dose calculation in the presence of respiratory motion. Int J Radiat Oncol Biol Phys. 2005;61 (5):1535-50. doi:10.1016/j. ijrobp.2004.11.037. Epub 2005/04/09.

26. Stroom JC, de Boer HC, Huizenga H, Visser AG. Inclusion of geometrical uncertainties in radiotherapy treatment planning by means of coverage probability. Int J Radiat Oncol Biol Phys. 1999;43(4):905-19. Epub 1999/03/31.

27. van Herk $M$, Remeijer $P$, Rasch $C$, Lebesque JV. The probability of correct target dosage: dose-population histograms for deriving treatment margins in radiotherapy. Int J Radiat Oncol Biol Phys. 2000;47(4):1121-35. Epub 2000/06/23.

28. Parker BC, Shiu AS, Maor MH, Lang FF, Liu HH, White RA, et al. PTV margin determination in conformal SRT of intracranial lesions. J Appl Clin Med Phys. 2002:3(3):176-89. doi:10.1120/1.1474308. Epub 2002/07/23.

29. Stevens CW, Munden RF, Forster KM, Kelly JF, Liao Z, Starkschall G, et al. Respiratory-driven lung tumor motion is independent of tumor size, tumor location, and pulmonary function. Int J Radiat Oncol Biol Phys. 2001;51 (1):62-8.

30. Guckenberger M, Meyer J, Wilbert J, Richter A, Baier K, Mueller G, et al. Intrafractional uncertainties in cone-beam $C T$ based image-guided radiotherapy (IGRT) of pulmonary tumors. Radiother Oncol. 2007;83(1):57-64. doi:10.1016/ j.radonc.2007.01.012. Epub 2007/02/20.

31. Worm ES, Hansen AT, Petersen JB, Muren LP, Praestegaard LH, Hoyer M. Inter- and intrafractional localisation errors in cone-beam $\mathrm{CT}$ guided stereotactic radiation therapy of tumours in the liver and lung. Acta Oncol. 2010;49(7):1177-83. doi:10.3109/0284186X.2010.498435. Epub 2010/07/02.

32. Rietzel E, Liu AK, Doppke KP, Wolfgang JA, Chen AB, Chen GT, et al. Design of $4 \mathrm{D}$ treatment planning target volumes. Int J Radiat Oncol Biol Phys. 2006; 66(1):287-95. doi:10.1016/j.jprobp.2006.05.024. Epub 2006/08/15.

33. Mori S, Endo M, Komatsu S, Yashiro T, Kandatsu S, Baba M. Four-dimensional measurement of lung tumor displacement using 256-multi-slice $\subset$ T-scanner. Lung Cancer. 2007;56(1):59-67. doi:10.1016/.jlungcan.2006.11.011. Epub 2006/12/13.

34. Descovich M, McGuinness C, Kannarunimit D, Chen J, Pinnaduwage D, Pouliot J, et al. Comparison between target margins derived from 4DCT scans and real-time tumor motion tracking: insights from lung tumor patients treated with robotic radiosurgery. Med Phys. 2015;42(3):1280-7. doi: 10.1118/1.4907956. Epub 2015/03/05.

35. Dobashi S, Sugane T, Mori S, Asakura H, Yamamoto N, Kumagai M, et al. Intrafractional respiratory motion for charged particle lung therapy with immobilization assessed by four-dimensional computed tomography. J Radiat Res. 2011;52(1):96-102. Epub 2011/02/05.

36. Timmerman R, Papiez L, McGarry R, Likes L, DesRosiers C, Frost S, et al. Extracranial stereotactic radioablation: results of a phase I study in medically inoperable stage I non-small cell lung cancer. Chest. 2003;124(5):1946-55. Epub 2003/11/08.

37. Lax I, Blomgren H, Naslund I, Svanstrom R. Stereotactic radiotherapy of malignancies in the abdomen. Methodological aspects. Acta Oncol. 1994; 33(6):677-83. Epub 1994/01/01

\section{Submit your next manuscript to BioMed Central and we will help you at every step:}

- We accept pre-submission inquiries

- Our selector tool helps you to find the most relevant journal

- We provide round the clock customer support

- Convenient online submission

- Thorough peer review

- Inclusion in PubMed and all major indexing services

- Maximum visibility for your research

Submit your manuscript at www.biomedcentral.com/submit
Biomed Central 